\title{
Single electron counting statistics for atto-Ampere currents through a semiconductor quantum dot
}

\author{
F. Hohls*, C. Fricke*, W. Wegscheider ${ }^{\dagger}$ and R. J. Haug* \\ *Institut für Festkörperphysik, Universität Hannover, Appelstr. 2, 30167 Hannover, Germany \\ ${ }^{\dagger}$ Angewandte und Experimentelle Physik, Universität Regensburg, D-93040 Regensburg, Germany
}

\begin{abstract}
We have implemented single electron counting for the tunneling current through a quantum dot. This allows us to study in detail the dynamics of transport. For certain settings of the gate and bias voltage we observe bimodal counting statistics which reveal the participation of different dot configurations in the transport process. The lifetime of these configurations is long compared to the mean tunneling times of the electrons.
\end{abstract}

Keywords: Quantum dot, Counting statistics, Fluctuations, Single electron counting PACS: $73.63 . \mathrm{Kv}, 73.23 . \mathrm{Hk}, 72.20 . \mathrm{My}$

Semiconductor quantum dots (QDs) are discussed as building block for devices with new functionalities, e.g. in the area of quantum information processing. This requires a thorough understanding of the dynamics of both of the quantum dots themselves and of the electron transport processes through them. It was shown that additional information beyond DC transport data can be gained by measurements of the shot noise of the transmitted current $[1,2,3]$ and of the third moment [4].

When interested in the regime of comparably low tunnel-coupling and small currents, which is the regime to be used for quantum information processing, one can use a different approach: Employing a quantum point contact (QPC) as charge detector near the quantum dot [5] it is possible to detect individual tunneling events onto and off a quantum dot [6]. This allows to measure the full counting statistics for unidirectional single electron transport through a QD [7] and, using a double dot system, can even be extended to bidirectional electron transport [8].

We use this method to examine the dynamics of single electron transport through a quantum dot for a wide range of gate and source-drain bias voltages [9]. For certain regimes which always involve accessible excited states in the QD we observe an interesting bimodal distribution of the counting distribution. We interpret this as the signature of different QD configurations involved in transport which have lifetimes in the order of several hundred millisecond.

The device used in this measurement is shown in Fig. 1a. The quantum dot is fabricated by local anodic oxidization, which allows to write insulating lines into a twodimensional electron system formed in a $\mathrm{GaAs} / \mathrm{Al}_{x} \mathrm{Ga}_{1-x} \mathrm{As}$ heterostructure [10, 11]. The QPC detector nearby allows the measurement of the QD charge state with a time resolution of $30 \mu \mathrm{s}$. This enables a time resolved analysis of individual tunneling events for currents through the QD that are in the order of atto-Ampere. 

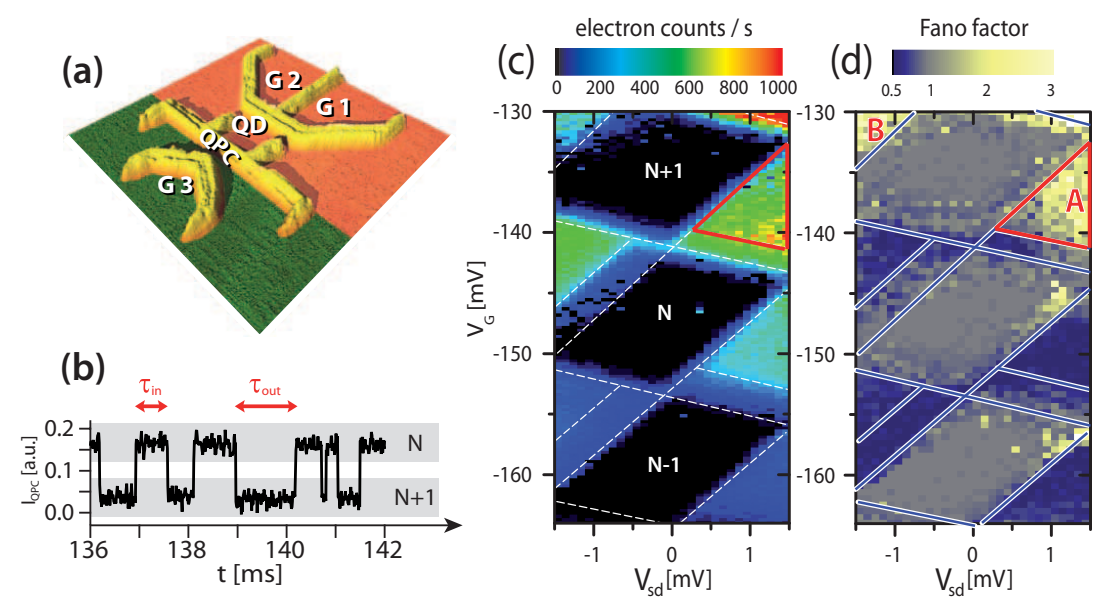

FIGURE 1. a) AFM micrograph of the device shows the quantum dot (QD) and the nearby charge detector (QPC). In-plane gates G1 and G2 control the quantum dot. b) Exemplary time trace of the charge signal $\left(V_{s d}=0.2 \mathrm{mV}\right)$. c) Electron counting rate as function of source-drain $\left(V_{s d}\right)$ and gate voltage $\left(V_{G}\right)$ exhibit Coulomb blockade diamonds. The lines denote changes of the average charge on the QD. d) Fano factor deduced from the counting distributions.

A typical time trace of the charge signal is shown in Fig. 1b. The signal switches between two levels which can be related to a different number of electrons on the QD. The trace was measured with a small bias of $0.2 \mathrm{mV}$ which ensures unidirectional transport: the transition $N \rightarrow N+1$ relates always to an electron tunneling from source onto the dot and $N+1 \rightarrow N$ to tunneling from the dot into the drain. The current $I$ thus is simply given bei the number of either of the two transitions per second multiplied by the elementary chare $e$. The counting rate shown in Fig. 1c is thus the equivalent of measuring the DC current through a quantum dot as function of bias and gate voltage. It reveals the usual Coulomb blockade diamonds of fixed electron number. The additional lines show changes of the mean charge on the dot and thereby reveal resonances of quantum dot states with the chemical potentials of either lead [12].

For each pair of $\left(V_{s d}, V_{G}\right)$ in Fig. 1c we measure a time trace of the QD charge with a length of $1 \mathrm{~s}$. The number of transitions yields the plotted electron count rate. For further statistical analysis we divide the trace into short segments and determine the number of transitions $n$ for each segment. This allows us now e.g. to determine the equivalent to the Fano factor which is used to characterize shot noise: $\alpha=\left\langle(n-\langle n\rangle)^{2}\right\rangle /\langle n\rangle$. The result is shown in Fig. 1d. We find that most of the time we observe $\alpha<1$ as expected for transport through a single level $[1,3]$ or for multiple level with the same outgoing rate. But there are some areas, e.g. the ones denoted by A and B, that show enhanced noise, $\alpha>1$.

Up to now we have only examined the mean value, i.e. the count rate (Fig. 1c), and the second moment (Fig. 1d) of the statistical distribution describing the dynamics of the single electron counting. But we measure the full counting distribution and will now make use of it to analyze the dynamics of the transport for regions of enhanced noise. 

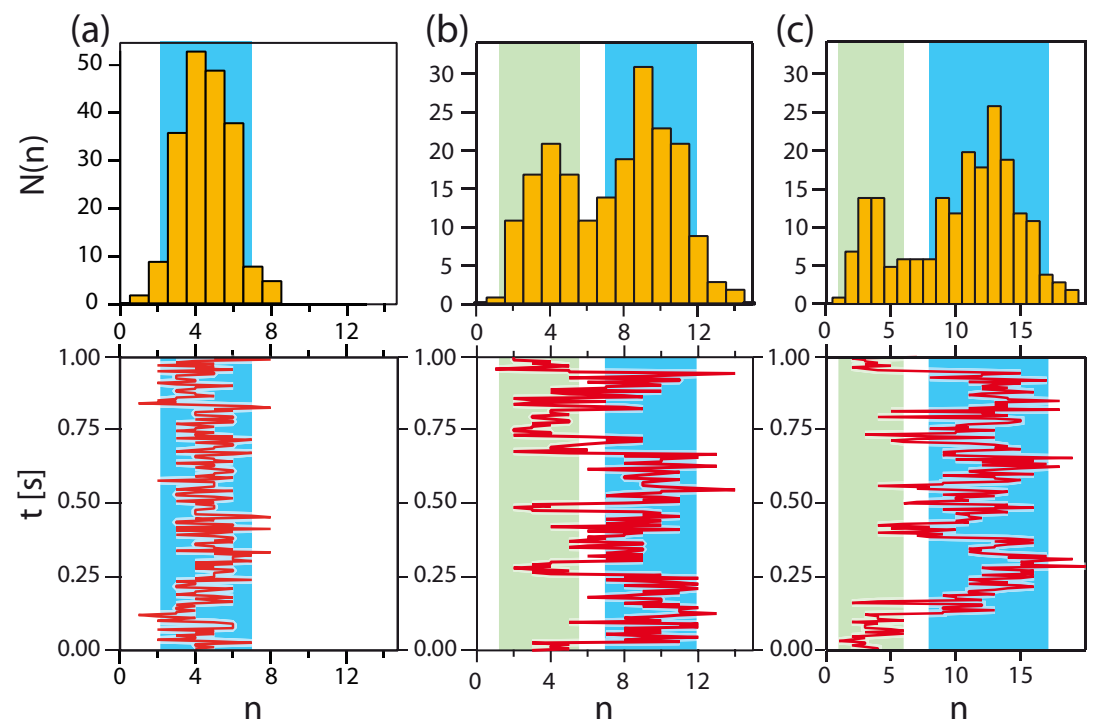

FIGURE 2. Counting distributions $N(n)$ (top) and time trace of the counting number $n$ (botttom) for ground state tunneling at small bias (a), for transport in region A of Fig. 1c (b), and for region B.

Fig. 2 displays the counting distribution for different settings of $\left(V_{s d}, V_{G}\right)$. These distributions show the number $N$ of the small analyzed segments that yield $n$ counts. For an infinite number of segments this distribution, divided by the number of segments, would converge towards the probability distribution of finding $n$ counts. Fig. 2a shows a typical distribution for tunneling through a single state on the dot. This situation is well understood and was examined theoretically $[13,14]$ and experimentally [7] with good agreement.

The distribution changes completely when analyzing the counting traces for the regions A and B in Fig. 1d where super-Poissonian noise is observed; Fig. 2b and Fig. 2c display the counting distributions for region A and B resp. We clearly observe a bimodal distribution with two distinct maxima. It is clear that this kind of distribution has a large width and therefore a large Fano factor.

For a better understanding of the underlying process we have examined the time evolution of the counting number $n$ per segment (lower part of Fig. 2). For tunneling through a single level (Fig. 2a) this time dependence is completely random and shows no further correlations. This is different for the case showing bimodal distribution (Fig. $2 \mathrm{~b}+\mathrm{c}$ ): Here the counting number per segment, $n$, stays for extended times of $\geq 100 \mathrm{~ms}$ in either of two bands that relate to the two peaks in the counting distribution.

The usual cycle for single electron tunneling reads as $(N) \rightarrow(N+1) \rightarrow(N)$ where $(N)$ and $(N+1)$ are the ground states for $N$ and $N+1$ electrons. For extended bias additional excited quantum dot states become accessible. E.g. a process $(N) \rightarrow(N+1)^{*}$ becomes possible where $(N+1)^{*}$ is an excited state with $N+1$ electrons. Usually this just leads to an increase of the tunneling current as it opens an extra channel but 
does not necessarily lead to super-Poissonian noise. Sometimes the rates involved in the $(N) \rightarrow(N+1)^{*} \rightarrow(N)$ channel are smaller than the one involving the $N+1$ electron ground state $(N+1)^{*}$. This can lead to super-Poissonian noise [15, 16] but concerning the distribution the effect will be just a broadening of the distribution and, depending on the rates and the chosen counting segments, possibly a large peak at $n=0$.

Therefore the bimodal distribution hints onto another process which we interpret as following: For certain QD configurations the transition from the excited $(N+1)^{*}$ state into the ground state $(N)$ might be much less likely than into an excited $(N)^{*}$ state. This initiates now a new transport cycle $(N)^{*} \rightarrow(N+1)^{*} \rightarrow(N)^{*}$ with tunneling rates that are somewhat different from the $(N) \rightarrow(N+1) \rightarrow(N)$ cycle. Thus transport through the QD happens via two different dot configurations with a slow transition on time scales $\geq 100 \mathrm{~ms}$ between them. While the one configuration is probably the ground state of the quantum dot, the likely cause for the second configuration is a long living charge excitation of an electron into a single particle state with low coupling to the leads when compared to the ones involved in the main single electron tunneling cycle [9].

In conclusion we have examined the counting statistics for single electron tunneling through a quantum dot. We found super-Poissonian noise which is related to bimodal counting distributions. The likely origin is a slow switching between two different dot configuration due to a long lived excitation within the dot.

\section{REFERENCES}

1. Y. M. Blanter, and M. Buttiker, Physics Reports 336, 1-166 (2000).

2. A. Nauen, I. Hapke-Wurst, F. Hohls, U. Zeitler, R. J. Haug, and K. Pierz, Physical Review B 66, 161303R (2002).

3. A. Nauen, F. Hohls, N. Maire, K. Pierz, and R. J. Haug, Physical Review B 70, 033305 (2004).

4. B. Reulet, J. Senzier, and D. E. Prober, Phys. Rev. Lett. 91, 196601 (2003).

5. M. Field, C. G. Smith, M. Pepper, D. A. Ritchie, J. E. F. Frost, G. A. C. Jones, and D. G. Hasko, Phys. Rev. Lett. 70, 1311 (1993).

6. J. M. Elzerman, R. Hanson, L. H. Willems van Beveren, B. Witkamp, L. M. K. Vandersypen, and L. P. Kouwenhoven, Nature 430, 431 (2004).

7. S. Gustavsson, R. Leturcq, B. Simovic, R. Schleser, T. Ihn, P. Studerus, K. Ensslin, D. C. Driscoll, and A. C. Gossard, Phys. Rev. Lett. 96, 076605 (2006).

8. T. Fujisawa, T. Hayashi, R. Tomita, and Y. Hirayama, Science 312, 1634 (2006).

9. C. Fricke, F. Hohls, W. Wegscheider, and R. J. Haug, to be published (2007).

10. R. Held, T. Vancura, T. Heinzel, K. Ensslin, M. Holland, and W. Wegscheider, Appl. Phys. Lett. 73, 262 (1998).

11. U. F. Keyser, H. W. Schumacher, U. Zeitler, R. J. Haug, and K. Eberl, Appl. Phys. Lett. 76, 457 (2000).

12. M. Rogge, B. Harke, C. Fricke, F. Hohls, M. Reinwald, W. Wegscheider, and R. J. Haug, Phys. Rev. Lett. 72, 233402 (2005).

13. L. S. Levitov, H. Lee, and G. B. Lesovik, J. Math. Phys. 37, 4845-4866 (1996).

14. D. A. Bagrets, and Y. V. Nazarov, Phys. Rev. B 67, 085316 (2003).

15. W. Belzig, Phys. Rev. B 71, 161301 (2005).

16. S. Gustavsson, R. Leturcq, B. Simovic, R. Schleser, P. Studerus, T. Ihn, K. Ensslin, D. C. Driscoll, and A. C. Gossard, Phys. Rev. B 74, 195305 (2006). 
Copyright of AIP Conference Proceedings is the property of American Institute of Physics and its content may not be copied or emailed to multiple sites or posted to a listserv without the copyright holder's express written permission. However, users may print, download, or email articles for individual use. 